\title{
Parámetros productivos y económicos de gallinas ponedoras ISA Brown en segundo ciclo de producción suplementadas con aminoácidos no esenciales
}

\author{
Productive ANd ECONOMIC PARAMETERS OF ISA Brown LAYING HENS IN THE SECOND \\ PRODUCTION CYCLE SUPPLEMENTED WITH NON-ESSENTIAL AMINO ACIDS
}

\author{
Walter Morales ${ }^{1}$, Victoria Rodríguez ${ }^{1,2}$, Noel Verjan ${ }^{1,2,3}$
}

\section{Resumen}

\begin{abstract}
El estudio buscó evaluar parámetros productivos y económicos en gallinas ponedoras de segundo ciclo Isa Brown suplementadas con glutamina + ácido glutámico $\left(\right.$ Aminogut $\left.{ }^{\circledR}\right)$. Se evaluaron cuatro niveles del producto comercial $(0,0.8,1.6$ y 2.4\%) en dietas para gallinas ponedoras de segundo ciclo durante trece semanas de producción (45-119 días). Se colectaron datos de desempeño productivo y se evaluaron parámetros económicos. La suplementación de la ración con Aminogut $₫$ al 1.6\% mejoró el número de huevos por ave alojada y la conversión alimenticia $(1.65 \mathrm{~kg}$ alimento por docena de huevos), aunque sin encontrar diferencias estadísticas para las variables consumo de alimento, tamaño del huevo, producción de huevos (\%) y masa de huevo. Por otro lado, la suplementación con Aminogut ${ }^{\circledR}$ durante las primeras dos semanas pos-muda, no obstante, constituye una estrategia para incrementar la producción de las aves y disminuir los costos de encasetamiento de nuevos lotes.
\end{abstract}

Palabras clave: alimentación; ácido glutámico; aves; glutamina; huevos

\section{AbSTRACT}

The objective of the study was to evaluate productive and economic parameters in Isa Brown laying hens of second cycle supplemented with glutamine + glutamic acid $\left(\right.$ Aminogut $\left.{ }^{\circledR}\right)$. Four levels of the commercial product $(0,0.8,1.6$ and $2.4 \%)$ were evaluated in diets for second cycle laying hens during thirteen weeks of production (45-119 days).

\footnotetext{
${ }^{1}$ Grupo de Investigación Avicultura, Laboratorio de Inmunología y Biología Molecular, Facultad de Medicina Veterinaria y Zootecnia, Universidad del Tolima, Colombia

${ }^{2}$ Grupo de Investigación en Inmunobiología y Patogénesis, Laboratorio de Inmunología y Biología Molecular, Facultad de Medicina Veterinaria y Zootecnia, Universidad del Tolima, Colombia

${ }^{3}$ E-mail:nverjang@ut.edu.co
}

Recibido: 24 de julio de 2017

Aceptado para publicación: 7 de marzo de 2018 
Productive performance data were collected, and economic parameters were evaluated. The supplementation of the ration with Aminogut ${ }^{\circledR}$ at $1.6 \%$ improved the number of eggs per housed bird and feed conversion (1.65 kg feed per dozen eggs), although without statistical differences for the variables food consumption, egg size, production of eggs (\%) and egg mass. On the other hand, supplementation with Aminogut ${ }^{\circledR}$ during the first two weeks would be a strategy to increase the production of birds and reduce the costs of encasing new lots.

Key words: eggs; food; glutamic acid; glutamine; poultry

\section{INTRODUCCIÓN}

La producción de huevo en Colombia obtuvo 12142 millones de unidades en 2015 y un crecimiento del 5.3\% con relación a 2014, principalmente debido al aumento del consumo interno, que pasó de 242 a 252 huevos por habitante (Ávila, 2015). Sin embargo, la industria avícola enfrenta fluctuaciones dramáticas en los costos de producción, en particular de las materias primas, donde los concentrados representan más del $70 \%$ de los costos variables, los cuales están sujetos a la fluctuación del tipo de cambio de las monedas duras (Ávila, 2015).

La muda forzada es una de las estrategias que permite reducir los costos de producción, dado que permite prolongar el periodo de vida útil de los animales y la mayor producción de huevos, generando ingresos adicionales sin la presión del encasetamiento de nuevos lotes (Park et al., 2004). No obstante, es poco lo que se conoce sobre los requerimientos nutricionales de las aves en un segundo ciclo de producción.

Las aves sujetas a muda forzada experimentan una regresión y atrofia severa de los tejidos, en particular una alteración en la integridad de la superficie de absorción intestinal que disminuye la capacidad de absorción y digestión (Ferrer et al., 1995; Macari, 1998; Pelicano et al., 2003; Maiorka, 2004; Zavarize et al., 2011). Los aminoácidos condicionalmente esenciales como la glutamina pueden ser sintetizados en cantidades adecuadas por los animales, pero necesitan ser suministrados en la dieta cuando las tasas de utilización tienen un incremento relativo a las tasas de síntesis ( $\mathrm{Neu}$ et al., 1996; Kim et al., 2007).

La glutamina y el ácido glutámico se consideran esenciales en estados hipercatabólicos como el destete (Texeira y Cardoso, 2011), injuria por ayuno, infecciones o enfermedad, oxidación y daño por endotoxinas (Mayorga, 2013; Ayazi, 2014). La glutamina constituye una fuente principal de energía para las células epiteliales del intestino delgado (Neu et al., 1996), favoreciendo el mayor número de células y del tamaño de las vellosidades (Macari, 1998; Maiorka, 2004; Carneiro et al., 2006), lo cual permitiría una mejor capacidad de absorción del intestino y mayor productividad de las aves.

La suplementación con glutamina en pollos de 0-28 días después de un ayuno de 48 horas pos-eclosión mejoró la eficiencia alimenticia, la morfología del intestino delgado y la respuesta a desafíos con Eimeria spp (Yi et al., 2005; Almeida et al., 2012; Sakamoto et al., 2014), así como una mejora en la ganancia de peso, conversión alimenticia y respuesta inmune (Soltan, 2009). La suplementación de pollos con $1 \%$ de glutamina y nucleótidos también mejoró los parámetros productivos y la morfología intestinal durante los primeros 21 días de vida de las aves (Zavarize et al., 2011). Por otro lado, Bartell y Batal (2007) observaron que la 
suplementación con $1 \%$ de glutamina incrementó en $11 \%$ la ganancia de peso en aves suplementadas durante 21 días, en tanto que Xiao-Ying et al. (2010) suplementando con $0.8 \%$ de glutamina a gallinas ponedoras mejoraron sus parámetros productivos. El presente estudio evaluó parámetros productivos y económicos en gallinas ponedoras ISA Brown de segundo ciclo de producción (posterior a la muda forzada) suplementadas con amino ácidos no esenciales (glutamina + ácido glutámico).

\section{Materiales y Métodos}

\section{Localización y Animales}

El experimento se realizó en la granja Avícola San Carlos, localizada en el municipio de San Pedro de los Milagros, a 2475 msnm y con $14{ }^{\circ} \mathrm{C}$ de temperatura promedio. El estudio con animales vivos fue aprobado por el Comité de Bioética de la Universidad del Tolima.

Se seleccionaron 400 gallinas ponedoras Isa Brown al final del primer ciclo de producción (76 semanas de edad) con pesos entre 1900 y $2000 \mathrm{~g}$ para iniciar un proceso de muda inducida. Las gallinas fueron encasetadas (4 aves por jaula) en 100 jaulas de alambre California, tipo piramidal, adecuadas para la alimentación de las aves y suministro de agua a través de bebederos de pitillo y pisos en malla.

\section{Muda Inducida}

El alimento fue retirado por 12 días, pero con disponibilidad permanente de agua para las aves. El momento en que las aves perdieron el $28 \%$ de su peso inicial se inició el suministro de granos de maíz partido durante nueve días (1-3: $30 \mathrm{~g}$; 4-6; $60 \mathrm{~g}$; 7-9: $90 \mathrm{~g} /$ ave/día), para luego proceder a una dieta de iniciación ( $21 \%$ proteína, $1 \%$ calcio) durante ocho días (10-17: $110 \mathrm{~g} / \mathrm{ave} /$ día), seguido de una dieta de levante (20\% proteína,
$2.5 \%$ calcio) durante nueve días (19-27: $115 \mathrm{~g} /$ ave/día), para finalmente suministrar alimento Prepico Producción ponedora Fase I (19\% proteína, $4.2 \%$ calcio) (día 27-119: $115 \mathrm{~g} /$ ave/día).

El retiro del alimento fue acompañado de una disminución a 9 horas luz y 15 horas de oscuridad por día, con una intensidad que se disminuyó de 100 a 20 lux en el día. Este periodo de menor luminosidad se reajustó a 14 horas de luz y 100 lux de intensidad promedio al día 42 de retiro del alimento. La producción de huevos durante la muda cesa totalmente el día 7 del retiro del alimento y se reinicia el día 29 del inicio del ayuno del ave.

\section{Preparación de las Dietas}

Las dietas fueron preparadas en la planta de alimentos del establecimiento. Las dietas fueron formuladas con base a maíz y torta de soya (Cuadro 1), según los requerimientos nutricionales de aves ponedoras (Rostagno et al., 2011). Los nutrientes aportados se indican en el Cuadro 2.

\section{Diseño Experimental}

Se usó un diseño completamente al azar con tres porcentajes $(0.8,1.6$ y $2.4 \%)$ de Aminogut $\AA$ y una dieta control libre del producto, para un total de cuatro tratamientos aplicados a 100 aves por grupo. El producto comercial Aminogut ${ }^{\circledR}$ (Ajinomoto Interamericana) es una asociación de glutamina (Gln) + acido glutámico (Glu) en concentración del 10\% (especificaciones del fabricante).

\section{Parámetros Productivos}

Las aves que obtuvieron una reducción del $28 \%$ de su peso inicial (532 a $560 \mathrm{~g}$ ) en el día 14 del ayuno iniciaron el consumo de maíz partido y a los nueve días se inició el suministro de las dietas suplementadas. Las aves fueron pesadas semanalmente hasta los 119 días de producción. Asimismo, la mortalidad de las aves fue registrada diariamente. 
Cuadro 1. Ingredientes $(\mathrm{kg} / \mathrm{t})$ en dietas prepico para gallinas ponedoras de segundo ciclo de producción ( 25 a 98 días)

\begin{tabular}{lcccc}
\hline \multirow{2}{*}{ Ingrediente } & \multicolumn{4}{c}{ Aminogut $(\%)$} \\
\cline { 2 - 5 } & $\mathrm{T} 1(0)$ & $\mathrm{T} 2(0.8)$ & $\mathrm{T} 3(1.6)$ & $\mathrm{T} 4(2.4)$ \\
\hline Aminogut ${ }^{\mathrm{N}}$ & 0 & 8 & 16 & 24 \\
Maíz amarillo & 561 & 567 & 574 & 580 \\
Torta de soya & 225 & 213 & 200 & 188 \\
Soya extruida & 70 & 70 & 70 & 70 \\
Harina de huesos calcinado & 7 & 7 & 7 & 7 \\
Aceite & 15 & 12 & 9 & 6 \\
Harina de pescado & 10 & 10 & 10 & 10 \\
Carbonato de calcio & 99 & 99 & 99 & 99 \\
Biomix Ponedora M.E. & 3 & 3 & 3 & 3 \\
DL metionina & 1.58 & 1.67 & 1.8 & 1.93 \\
Sal de mar & 3.67 & 3.65 & 3.53 & 3.4 \\
Toxibón & 2 & 2 & 2 & 2 \\
Lisina HCl & 1.28 & 1.68 & 2.07 & 2.47 \\
S- bicarbonato & 0 & 0 & 0.14 & 0.27 \\
Treonina & 0.71 & 0.89 & 1.08 & 1.27 \\
Inhisalm & 0.5 & 0.5 & 0.5 & 0.5 \\
Triptófano & 0.04 & 0.11 & 0.17 & 0.24 \\
\hline Total & 999.78 & 999.5 & 999.29 & 999.08 \\
\hline
\end{tabular}

La producción de huevos se inició en el día 17 de la reanudación del consumo de alimento. La producción de huevo se registró diariamente y por grupos hasta el día 119. Los huevos fueron recogidos dos veces al día. Doce huevos por tratamiento fueron pesados semanalmente en una báscula Hopex EK3130-05 (Cali, Colombia) con graduación en gramos. La masa de huevos se obtuvo de multiplicar la producción diaria por el peso de cada tipo de huevo producido en el día. Se calculó, además, el número de huevos por ave alojada, lo cual corresponde al número de huevos que produce un ave desde el momento de ser alojada en producción. Se evaluó semanalmente tomando la producción semanal de huevos, dividida entre el número inicial de aves de cada tratamiento. La conversión alimenticia se evaluó semanalmente toman- do el consumo de alimento en gramos y dividiéndolo entre la docena de huevos para cada tratamiento.

Los huevos fueron clasificados por peso según la segunda actualización de la Norma Técnica Colombiana NTC (ICONTEC) 1240. Se utilizaron las siguientes categorías: Jumbo (>78 g); AAA (67-77.9 g); AA (60$66.8 \mathrm{~g})$; A (53-59.9 g); B (46-52.9 g), C (>46 g) (https://vdocuments.site/ntc-1240huevospdf).

\section{Análisis Económico}

Para el análisis económico se consideraron los siguientes costos: el costo por kilogramo de alimento promedio durante las tres fases de alimentación fue de $\$ 1000$ pesos 
Cuadro 2. Nutrientes (\%) en dietas prepico para gallinas ponedoras de segundo ciclo de producción ( 25 a 98 días)

\begin{tabular}{lcccc}
\hline \multirow{2}{*}{ Ingrediente } & Control & \multicolumn{3}{c}{ Aminogut $(\%)$} \\
\cline { 2 - 5 } & $\mathrm{T} 1$ & $\mathrm{~T} 2$ & $\mathrm{~T} 3$ & $\mathrm{~T} 4$ \\
\hline Energía Met* (kcal/kg) & 2900 & 2900 & 2900 & 2900 \\
Proteína bruta (\%) & 19 & 19 & 19 & 19 \\
Calcio (\%) & 4.2 & 4.2 & 4.2 & 4.2 \\
Fósforo disponible (\%) & 0.34 & 0.34 & 0.34 & 0.34 \\
Met + Cis** digestible (\%) & 0.71 & 0.71 & 0.71 & 0.71 \\
Metionina digestible (\%) & 0.44 & 0.44 & 0.45 & 0.46 \\
Lisina digestible (\%) & 1 & 1 & 1 & 1 \\
Arginina digestible (\%) & 1.12 & 1.08 & 1.05 & 1.01 \\
Treonina digestible (\%) & 0.71 & 0.71 & 0.71 & 0.71 \\
Triptófano digestible (\%) & 0.2 & 0.2 & 0.2 & 0.2 \\
Sodio (\%) & 0.18 & 0.18 & 0.18 & 0.18 \\
Cloro (\%) & 0.29 & 0.3 & 0.3 & 0.3 \\
Potasio (\%) & 0.77 & 0.74 & 0.72 & 0.69 \\
BED*** (mEq/kg) & 198 & 192 & 188 & 184 \\
\hline
\end{tabular}

* Metabolizable; **Metionina + Cistina; ***BED: Balance electrolítico de la dieta

colombianos, el costo de alimentación por kilogramo de alimento durante las tres fases de alimentación varió entre \$10407 y \$10749. El número de huevos producidos varió entre 470 y 521 docenas. El costo de alimento por docena de huevos varió entre \$1665 y \$1771.

El ingreso bruto resulta de la cantidad de huevos producidos multiplicado por el precio de venta al momento de la investigación (este valor varió entre $\$ 1240800$ y \$1 374120 entre tratamientos). El margen bruto indica la diferencia entre el ingreso bruto y el costo total de la docena de huevos, el cual varió entre \$130619 y \$ 186384 entre tratamientos. El margen bruto relativo es el valor porcentual del margen bruto (varió entre 10.2 y $14.2 \%$ ).

\section{Análisis Estadístico}

Los datos obtenidos fueron sometidos al análisis de varianza para determinar dife- rencias entre tratamientos. Se calculó el valor $\mathrm{F}$ y a aquellos con un valor $\mathrm{p}<0.05$ se les aplicó la prueba de rangos múltiples de Duncan para establecer diferencias entre ellos. Los análisis estadísticos se realizaron con el programa SPSS v. 20.

\section{Resultados}

El comportamiento productivo de gallinas ponedoras Isa Brown de segundo ciclo suplementadas con Aminogut ${ }^{\circledR}$ durante 119 días reveló un consumo de alimento entre 113 y 119 g/ave/día, siendo mayor en T3 (118.9 $\mathrm{g} / \mathrm{ave} / \mathrm{dí} a ; \mathrm{p}<0.05)$. El porcentaje de producción de huevos estuvo entre 61.98 y $68.64 \%$, sin haber diferencias significativas entre tratamientos (Cuadro 3).

La conversión alimenticia por docena de huevo estuvo entre 1.65 y 1.82 , sin diferencia significativa entre tratamientos; en tanto 
Cuadro 3. Parámetros productivos de gallinas Isa Brown en segundo ciclo de producción, suplementadas con Aminogut ${ }^{\circledR}$ hasta al día 119 desde el inicio de la muda

\begin{tabular}{lcccc}
\hline Parámetro & $\mathrm{T} 1$ & $\mathrm{~T} 2$ & $\mathrm{~T} 3$ & $\mathrm{~T} 4$ \\
\hline Consumo de alimento $(\mathrm{g})$ & $114.36^{\mathrm{a}}$ & $113.83^{\mathrm{a}}$ & $118.9^{\mathrm{b}}$ & $113.75^{\mathrm{a}}$ \\
Producción de huevos $(\%)$ & $63.42^{\mathrm{a}}$ & $61.98^{\mathrm{a}}$ & $63.95^{\mathrm{a}}$ & $68.64^{\mathrm{a}}$ \\
Peso de huevos $(\mathrm{g})$ & $62.67^{\mathrm{a}}$ & $61.14^{\mathrm{a}}$ & $67.49^{\mathrm{a}}$ & $63.24^{\mathrm{a}}$ \\
Conversión alimenticia & $1.79^{\mathrm{a}}$ & $1.81^{\mathrm{a}}$ & $1.65^{\mathrm{a}}$ & $1.82^{\mathrm{a}}$ \\
Mortalidad (\%) & 0 & 0 & 0 & 0 \\
Masa de huevos & $42.60^{\mathrm{a}}$ & $40.97^{\mathrm{a}}$ & $46.05^{\mathrm{a}}$ & $42.29^{\mathrm{a}}$ \\
Huevos por ave & $4.63^{\mathrm{ab}}$ & $4.52^{\mathrm{a}}$ & $5.01^{\mathrm{c}}$ & $4.68^{\mathrm{b}}$ \\
alojada/semana & & & \\
\hline
\end{tabular}

$a, b, c$ Letras diferentes para la misma línea denotan diferencias al nivel de $p<0.05$

Cuadro 4. Número de huevos y distribución porcentual por tratamiento, según tamaño de huevo

\begin{tabular}{cccccccc}
\hline $\mathrm{T}^{1}$ & Jumbo & AAA & AA & A & B & C & Total \\
\hline 1 & 90 & 2028 & 2056 & 309 & 0 & 0 & 4483 \\
& $(0.49 \%)$ & $(11.02 \%)$ & $(11.18 \%)$ & $(1.68 \%)$ & & & $(24.37 \%)$ \\
2 & 32 & 1803 & 2337 & 400 & 0 & 0 & 4572 \\
& $(0.17 \%)$ & $(9.81 \%)$ & $(12.71 \%)$ & $(2.17 \%)$ & & & $(24.86 \%)$ \\
3 & 40 & 2584 & 2027 & 292 & 0 & 0 & 4943 \\
& $(0.22 \%)$ & $(14.05 \%)$ & $(11.02 \%)$ & $(1.59 \%)$ & & & $(26.87 \%)$ \\
4 & 46 & 1705 & 2257 & 387 & 0 & 0 & 4395 \\
& $(0.25 \%)$ & $(9.27 \%)$ & $(12.27 \%)$ & $(2.10 \%)$ & & & $(23.89 \%)$ \\
\hline Total & 208 & 8120 & 8677 & 1388 & 0 & 0 & 18393 \\
& $(1.13 \%)$ & $(44.15 \%)$ & $(47.17 \%)$ & $(7.55 \%)$ & & & $(100 \%)$ \\
\hline
\end{tabular}

${ }^{1}$ Dietas a base a maíz y torta de soya, suplementadas con T1: 0, T2: 0.8, T3: 1.6, T4: 2.4\% de Aminogut ${ }^{\circledR}$

Cuadro 5. Parámetros económicos (pesos colombianos) de gallinas Isa Brown en segundo ciclo de producción suplementadas con Aminogut ${ }^{\circledR}$ al día 119 desde el inicio de la muda

\begin{tabular}{|c|c|c|c|c|c|c|c|c|c|}
\hline 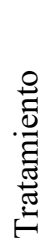 & 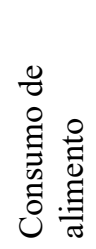 & $\begin{array}{l}\frac{\pi}{0} \\
\stackrel{\theta}{0} \\
0 \\
0 \\
0 \\
0\end{array}$ & 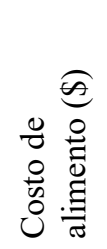 & $\begin{array}{l}{ }_{0}^{\infty} \\
0 \\
0 \\
0 \\
0\end{array}$ & 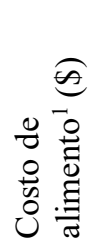 & $\begin{array}{l}\overparen{E} \\
-0 \\
i \stackrel{0}{0} \\
0 \\
0 \\
0 \\
0\end{array}$ & 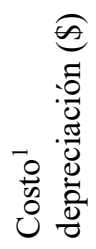 & 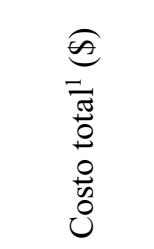 & 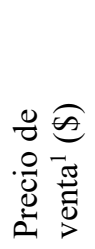 \\
\hline $\mathrm{T} 1$ & 10.41 & 1000.0 & 10407 & 481 & 1665 & 240 & 360 & 1089081 & 2640 \\
\hline $\mathrm{T} 2$ & 10.36 & 1012.8 & 10491 & 470 & 1739 & 240 & 360 & 1099311 & 2640 \\
\hline T3 & 10.82 & 1025.6 & 11097 & 521 & 1682 & 240 & 360 & 1187736 & 2640 \\
\hline $\mathrm{T} 4$ & 10.35 & 1038.4 & 10749 & 485 & 1771 & 240 & 360 & 1149561 & 2640 \\
\hline
\end{tabular}

${ }^{1}$ Por docena de huevos 
Cuadro 6. Margen de ganancia (pesos colombianos) en gallinas Isa Brown en segundo ciclo de producción suplementadas con Aminogut ${ }^{\circledR}$

\begin{tabular}{|c|c|c|c|}
\hline 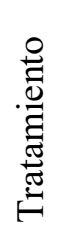 & 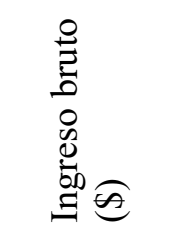 & 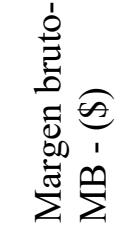 & 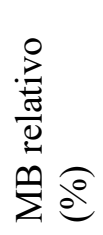 \\
\hline $\mathrm{T} 1$ & 1269620 & 180539 & 14.2 \\
\hline $\mathrm{T} 2$ & 1240800 & 141489 & 11.4 \\
\hline T3 & 1374120 & 186384 & 13.6 \\
\hline $\mathrm{T} 4$ & 1280180 & 130619 & 10.2 \\
\hline
\end{tabular}

Cuadro 7. Margen de ganancia (pesos colombianos) en gallinas Isa Brown en segundo ciclo de producción suplementadas con Aminogut ${ }^{\circledR}$ durante las dos primeras semanas

\begin{tabular}{|c|c|c|c|}
\hline 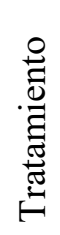 & 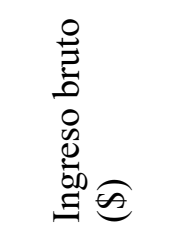 & 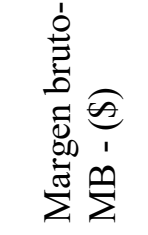 & 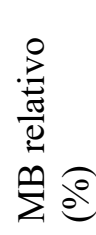 \\
\hline $\mathrm{T} 1$ & 1269620 & 180359 & 14.2 \\
\hline $\mathrm{T} 2$ & 1240800 & 158826 & 12.8 \\
\hline $\mathrm{T} 3$ & 1374120 & 223119 & 16.2 \\
\hline $\mathrm{T} 4$ & 1280180 & 183253 & 14.3 \\
\hline
\end{tabular}

que el número de huevos por ave alojada semanal fue entre 4.52 y 5.01 , siendo mayor en $\mathrm{T} 3$ y menor en T2 $(\mathrm{p}<0.05)$. Por otro lado, la masa de huevos estuvo entre 40.97 y 46.05 $\mathrm{g} /$ huevo, no encontrándose diferencia significativa entre tratamientos. No se registró mortalidad alguna durante el estudio (Cuadro 3 ).
El tamaño de huevo presentó variaciones según el tratamiento, siendo AAA y AA los tamaños con porcentajes de mayor producción. Los huevos AA presentaron el mayor porcentaje de producción en total (47.17\%), especialmente en T2. La mayor cantidad de huevos AAA producidos se observó en T3 (14.05\%), siendo el porcentaje más alto por tratamiento y el tratamiento con mayor producción de huevos $(26.87 \%)$. T4 registró la menor cantidad de huevos para todos los tamaños $(23.89 \%)$. No se obtuvieron huevos tamaño $\mathrm{B}$ y $\mathrm{C}$ durante el periodo de experimentación (Cuadro 4).

La suplementación durante las fases de iniciación, levante y producción de segundo ciclo no mejoró el margen relativo bruto (Cuadros 5 y 6), indicando que el uso del Aminogut ${ }^{\circledR}$ en estas dietas no es económicamente viable. Sin embargo, el análisis económico durante las dos primeras semanas de alimentación muestra que T3 (1.6\% Aminogut $\left({ }^{\circledR}\right)$ presenta el mayor margen bruto relativo (Cuadro 7).

\section{Discusión}

El Aminogut $\AA$ es un compuesto que aporta los aminoácidos glutamina y ácido glutámico, los cuales han sido asociados a la recuperación y proliferación del epitelio intestinal después de la muda forzada en aves ponedoras (Maiorka, 2004; Xiao-Ying et al., 2010). Los resultados demuestran que las aves ponedoras comerciales Isa Brown de segundo ciclo de producción suplementadas con $1.6 \%$ de Aminogut ${ }^{\circledR}$ incrementaron el número de huevos por ave alojada y lograron una mayor eficiencia de conversión alimenticia, en concordancia con Xiao Ying et al. (2010), quienes documentaron que la suplementación con glutamina en la dieta mejora la conversión alimenticia y el consumo de alimento en gallinas ponedoras de líneas semipesadas en primer ciclo de producción. No obstante, el análisis económico mostró que la suplementación con Aminogut ${ }^{\circledR}$ 
durante todo el ciclo productivo no es económicamente viable y que, por el contrario, se podrían obtener efectos positivos en términos económicos con solo suplementar en las dos primeras semanas posteriores a la muda.

El número de huevos por ave alojada por semana indicó una mejor respuesta en el grupo T3 (5.01 huevos) comparado con el grupo control (4.63). Estos resultados son superiores a los registrados en el manual HyLine (2016) de la línea de aves del estudio, que registra 4.74 huevos por semana y los hallados por Mazzuco et al. (2011) en aves HyLine W-36 de segundo ciclo productivo (4.55) durante 56 semanas de producción. Los resultados sugieren que este parámetro productivo es susceptible de ser mejorado en gallinas ponedoras Isa Brown de segundo ciclo.

La suplementación con glutamina + ácido glutámico en gallinas ponedoras de segundo ciclo mostró un efecto positivo en la conversión alimenticia por docena de huevo, la suplementación con glutamina + ácido glutámico al $1.6 \%$ en las dietas de producción obtuvieron una conversión de $1.65 \mathrm{~kg}$ por docena de huevo, siendo la mejor entre los grupos, pero sin presentar diferencias significativas (Cuadro 3). Estos resultados difieren de los hallados por Sgavioli et al. (2013) en aves Isa Brown de 71 semanas de edad durante 112 días de producción pos-muda, quienes registraron una conversión de $1.77 \mathrm{~kg}$ de alimento por docena de huevo. Adicionalmente, la conversión alimenticia obtenida en el grupo suplementado con Aminogut ${ }^{\circledR}$ al $1.6 \%$ fue superior a la reportada en aves Shaver (1.79) de 70 semanas de edad durante 112 días de producción postmuda (Molino et al., 2009).

La suplementación con glutamina + ácido glutámico al $1.6 \%$ afectó el consumo de alimento, habiendo diferencias significativas entre tratamientos. El mayor consumo fue en T3 (118.9 g/ave/día, y el menor consumo en T4 (113.75 g/ave/día). Estos resultados son superiores a los $115.4 \mathrm{~g} /$ ave/día reportados en aves Hissex Brown de 100 semanas de edad durante 112 días pos-muda (Girardon, 2011), y a los $104 \mathrm{~g}$ /ave/día reportados por Sgavioli et al. (2013) en aves Isa Brown de 71 semanas de edad durante 112 días de producción pos-muda.

El peso de los huevos no fue afectado por los tratamientos del estudio (61.14-67.49 g), resultados similares a los documentados en el manual Hyline Brown con registros de $65.21 \mathrm{~g}$ en aves de segundo ciclo durante 266 días de producción pos-muda (Keshavarz y Quimby, 2002), así como a los trabajos de Molino et al. (2009) en aves Shaver de 70 semanas de edad sometidas a muda inducida tradicional y 140 días de producción posmuda, y de Girardon (2011) en aves Hissex Brown de 100 semanas de edad sometidas a muda inducida y 142 días de producción.

La masa de los huevos (40.97 y $46.05 \mathrm{~g}$ ) tampoco fue afectada por la suplementación con Aminogut $\AA$. Los resultados coinciden con los trabajos de Mejía et al. (2011) en aves Hy-Line W-36 de 69 semanas de edad (45.2 g) sometidas a muda inducida y 245 días de producción pos-muda, y de Keshavarz y Quimby (2002) en aves Babcock B300 de 66 semanas de edad (46.4 g) durante 196 días de producción pos-muda. No obstante, los resultados de este estudio fueron inferiores a los 50.12 g hallados por Sgavioli et al. (2013) en aves de huevo marrón Isa Brown de 71 semanas de edad sometidas a muda inducida y 112 días de producción pos-muda. En conjunto, estos estudios sugieren un posible efecto de la línea genética de las gallinas sobre la masa de los huevos.

La producción de huevos en este estudio estuvo entre 61.98 y $68.64 \%$, sin diferencia significativa entre tratamientos, e inferior al $72.74 \%$ registrada en aves de huevo color marrón Isa Brown de 72 semanas de edad sometidas a muda inducida (Sgavioli et al., 2013), al 78.08\% en aves Hissex Brown de 100 semanas de edad sometidas a muda in- 
ducida durante 112 días de producción posmuda (Girardon, 2011) y al $71.4 \%$ en aves $\mathrm{H} \& \mathrm{~N}$ de 95 semanas de edad sometidas a muda inducida y en 140 días de producción pos-muda (Gongruttananun et al., 2013). Estas diferencias podrían ser atribuidas a las diferencias genéticas, la calidad nutricional y bromatológica del alimento, el estatus sanitario de las granjas, planes vacunales, y calidad de las vellosidades intestinales (Hy-Line, 2016).

Finalmente, es de resaltar que en este estudio no se observaron casos de mortalidad. Esta puede llegar a $6.7 \%$ en aves de huevo color marrón Hyline Brown sometidas a muda inducida (Hy-Line, 2016). La mortalidad en aves de segundo ciclo fluctúa entre $0.2 \%$ en aves Shaver de 70 semanas de edad (Molino et al., 2009), y 2\% en aves Hy line W-36 de 65 semanas de edad sometidas a muda inducida y 280 días de producción posmuda (Webster, 2000).

\section{Conclusiones}

- La suplementación con Aminogut ${ }^{\circledR}$ a dosis de $1.6 \%$ en dietas de gallinas ponedoras Isa Brown de segundo ciclo de producción constituye una estrategia que podría mejorar la conversión alimenticia por docena de huevo y el número promedio de huevos por ave alojada por semana.

- La suplementación con Aminogut ${ }^{\circledR}$ al $0.8 \%$ y $2.4 \%$ no mostró efectos positivos en dichos parámetros productivos y aparentemente podrían tener efectos imperceptibles o deletéreos en las aves, respectivamente.

- La suplementación con Aminogut ${ }^{\circledR}$ durante el ciclo productivo no fue económicamente viable; no obstante, se podrían obtener efectos positivos en términos económicos con solo suplementar en las dos primeras semanas posteriores a la muda.

\section{Agradecimientos}

Los autores expresan su agradecimiento a la Oficina Central de Investigaciones de la Universidad del Tolima por el financiamiento de esta investigación (Proyecto 250114), así como a las directivas y personal de la empresa avícola San Carlos en San Pedro de los Milagros por las facilidades prestadas para el desarrollo de la investigación.

\section{Literatura Citada}

1. Almeida RR, Ramos FS, Minafra CS, Duarte EF, Silva JMS, García WJ. 2012. Desenvolvimento digestivo de frangos de corte de crescimento lento e rápido. I Congreso de Pesquisa e Posgraduacao. Instituto Federal de Educacao, Ciencia y Tecnología, Goias, Goiânia, Brasil.

2. Ávila F. 2015. Avicultura con buena calificación. Avicultores 223: 18-37.

3. Ayazi M. 2014. The effect of dietary glutamine supplementation on performance and blood antioxidant status of broiler chickens under continuous heat stress condition. Intl J Farm Alli Sci 3: 12131219.

4. Bartell SM, Batal AB. 2007. The effect of supplemental glutamine on growth performance, development of the gastrointestinal tract, and humoral immune response of broilers. Poult Sci 86: 1940-194. doi: 10.1093/ps/86.9.1940

5. Carneiro BA, Fujii J, Brito GA, Alcantara C, Oriá RB, Lima AA, Obrig T, Guerrant RL. 2006. Caspase and bid involvement in Clostridium difficile toxin A-induced apoptosis and modulation of toxin A effects by glutamine and alanyl-glutamine in vivo and in vitro. Infect Immun 74: 81-87. doi: 10.1128/IAI.74.1.81-87.2006

6. Ferrer R, Planas JM, Moretó M. 1995. Cell apical surface area in enterocytes from chicken small and large 
intestine during development. Poult Sci 74: 1995-2002. doi: 10.3382/ps.0741995

7. Girardon JC. 2011. Métodos nutricionais de muda forçada em poedeiras semipesadas. Tesis de Maestría. Rio Grande do Sul, Brazil: Universidade Federal de Pelotas. 82 p.

8. Gongruttananun N, Boonkaewnan C, Lungyai V, Guntapa P. 2013. Utilization of different basal diets for molt induction in a strain of commercial laying hens. Kasetsart J 47: 205-216.

9. Hy-Line. 2016. Ponedoras comerciales Hy-Line Brown. Guía de manejo. [Internet]. Available in: http// www.hyline.com/UserDocs/Pages/ BRN_COM_SPN.pdf

10. Keshavarz $\bar{K}$, Quimby FW. 2002. An investigation of different molting techniques with an emphasis on animal welfare. J Appl Poultry Res 11: 54-67. doi: 10.1093/japr/11.1.54

11. Kim SW, Mateo RD, Yin YL, Wu G. 2007. Functional amino acids and fatty acids for enhancing production performance of sows and piglets. Asian Aust $\mathrm{J}$ Anim Sci 20: 295-306. doi: 10.5713/ ajas.2007.295

12. Macari M. 1998. Aspectos fisiológicos do sistema digestivo das aves. En: VIII SACAVET. Sao Paulo, Brasil.

13. Maiorka A. 2004. Impacto da saude intestinal na produtividade avícola. En: $\mathrm{V}$ Simposio Brasil Sul de Avicultura. Universidade Federal Do Parana, Brasil.

14. Mayorga M. 2013. Glutamina y salud intestinal de monogástricos. Plumazos 46: 4-12.

15. Mazzuco H, Avila VS, Coldebella A, Mores R, Jaenisch FRF, Lopes LS. 2011. Comparison of the effect of different methods of molt: production and welfare evaluation. Poult Sci 90: 29132920. doi: 10.3382/ps.2011-01670

16. Mejía L, Meyer ET, Studer DL, Utterback PL, Utterback CW, Parsons CM, Koelkebeck KW. 2011. Evaluation of limit feeding varying levels of distillers dried grains with solubles in non-feed- withdrawal molt programs for laying hens. Poult Sci 90: 321-327. doi: 10.3382/ ps.2010-01078

17. Molino AB; García EA, Berto DA, Pelicia K, Silva AP, Vercese F. 2009.

The effects of alternative forced-molting methods on the performance and egg quality of commercial layers. Rev Bras Ciên Avíc 11: 109-113. doi: 10.1590/ S1516-635X2009000200006

18. Neu J, Shenoy V, Chakrabarti R. 1996. Glutamine nutrition and metabolism: where do we go from here? FASEB J 10: 829-837.

19. Park SY, Kim WK, Birkhold SG, Kubena LF, Nisbet DJ, Rickel. 2004. Induced moulting issues and alternative dietary strategies for the egg industry in the United States. World Poultry Sci J 60: 196-209. doi: 10.1079/WPS200313

20. Pelicano ERL, de Souza PA, de Souza HBA, Oba A, Norkus EA, Kodawara LM, de Lima TMA. 2003. Morfometria e ultra-estrutura da mucosa intestinal de frangos de corte alimentados com dietas contendo diferentes probióticos. Rev Port Ciên Vet 98: 125-134.

21. Rostagno HS, Albino LFT, Donzele $J L$, Gomes PC, Oliveira RF, Lopes DC, Ferreira AS, et al. 2011. Tablas brasileras para aves y cerdos: composición de alimentos y requerimientos nutricionales. $3^{\circ}$ ed. Brasil: Universidad Federal de Vicosa. 259 p.

22. Sakamoto MI, Faria DE, Nakagi VS, Muarakami AE. 2014. Sources of trophic action on performance and intestinal morphometry of broiler chickens vaccinated against coccidisis. Rev Bras Ciên Avíc 16: 389-396. doi: 10.1590/ 1516-635X1604389-396

23. Sgavioli S, Filardi R, Praes MFFM, Domingues CH, Andrade P, Boleli IC, Junqueira OM. 2013. Effect of forcedmolting methods and rearing temperatures on the performance and organ biometrics of laying hens. Rev Bras Ciên Avíc 15: 239-245. doi: 10.1590/ S1516-635X2013000300011 
24. Soltan MA. 2009. Influence of dietary glutamine supplementation on growth performance, small intestinal morphology, immune response and some blood parameters of broiler chicken. Int J Poult Sci 8: 60-68. doi: 10.3923/ijps.2009.60.68

25. Teixeira RSC, Cardoso WM. 2011. Muda forcada na avicultura moderna. Rev Bras Reprod Anim 45: 444-455.

26. Webster AB. 2000. Behavior of White Leghorn laying hens after withdrawal of feed. Poult Sci 79: 192-200.

27. Xiao-Ying D, Chu-Fen Y, Sheng-Qui T, Qing-Yan J, Xiao-Ting Z. 2010. Effect and mechanism of glutamine on productive performance and egg quality of laying hens. Asian Aust J Anim Sci 23: 1049-1056. doi: 10.5713/ajas.2010.90611

28. Yi FG, Allee GL, Knight CD, Dibner JJ. 2005. Impact of glutamine and oasis hatchling supplement on growth performance, small intestinal morphology, and immune response of broilers vaccinated and challenged with Eimeria maxima. Poult Sci 84: 283-293.

29. Zavarize KC, Sartori JR, Pelícia VC, Pezzato AC, Araujo PC, Stradiotti AC, Madeira LA. 2011. Glutamina e nucleotídeos na dieta de frangos de corte criados no sistema alternativo. Arch Zootec 60: 913-920. doi: 10.4321/S000405922011000400008 